\title{
Detection of Closed Sharp Feature Lines in Point Clouds for Reverse Engineering Applications
}

\author{
Kris Demarsin \\ Denis Vanderstraeten \\ Tim Volodine \\ Dirk Roose \\ Report TW 458, May 2006
}

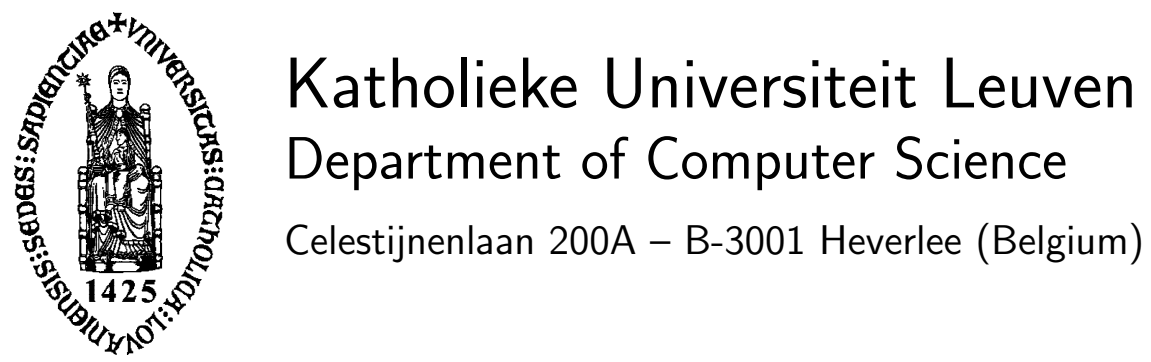




\title{
Detection of Closed Sharp Feature Lines in Point Clouds for Reverse Engineering Applications
}

\author{
Kris Demarsin \\ Denis Vanderstraeten \\ Tim Volodine \\ Dirk Roose \\ Report TW 458, May 2006
}

Department of Computer Science, K.U.Leuven

\begin{abstract}
The reconstruction of a surface model from a point cloud is an important task in the reverse engineering of industrial parts. We aim at constructing a curve network on the point cloud that will define the border of the various surface patches. In this paper, we present an algorithm to extract closed sharp feature lines, which is necessary to create such a closed curve network. We use a first order segmentation to extract candidate feature points and process them as a graph to recover the sharp feature lines. To this end, a minimum spanning tree is constructed and afterwards a reconnection procedure closes the lines. The algorithm is fast and gives good results for real-world point sets from industrial applications.
\end{abstract}

Keywords : sharp edges, point clouds, segmentation.

CR Subject Classification : I.3.5, G.2.2

AMS(MOS) Classification : Primary : 65D18, Secondary : 68R10. 


\title{
Detection of Closed Sharp Feature Lines in Point Clouds for Reverse Engineering Applications
}

\author{
Kris Demarsin, Denis Vanderstraeten† Tim Volodine, Dirk Roose
}

May 11, 2006

\begin{abstract}
The reconstruction of a surface model from a point cloud is an important task in the reverse engineering of industrial parts. We aim at constructing a curve network on the point cloud that will define the border of the various surface patches. In this paper, we present an algorithm to extract closed sharp feature lines, which is necessary to create such a closed curve network. We use a first order segmentation to extract candidate feature points and process them as a graph to recover the sharp feature lines. To this end, a minimum spanning tree is constructed and afterwards a reconnection procedure closes the lines. The algorithm is fast and gives good results for real-world point sets from industrial applications.
\end{abstract}

\section{Introduction}

Feature lines can be mathematically defined via local extrema of principal curvatures along corresponding principal directions. These feature lines can be used for visualization purposes: point clouds are visually easier to understand if the feature lines are highlighted in the visualization. In addition, the quality of a mesh can be improved when the feature lines are known. Shape recognition and quality control are other application areas of feature line extraction.

Many feature line extraction algorithms rely on a triangular mesh as input, e.g. [3], [5], [6], [8], and [13]. Few algorithms only use a point cloud, e.g. [2] and [7]. However, these existing methods usually result in pieces of unconnected feature lines, making it hard to segment a point cloud or mesh into surface patches, based on these lines.

Since we aim at constructing a curve network on a point cloud, that will define the border of the various surface patches, this paper focusses on finding closed sharp feature lines. We use a region growing method, which is a modification of the method of Vanco et al. ([9], [10], and [11]), to segment a point cloud in clusters of points (segments) and to detect sharp edges. We build and manipulate a graph of these segments, resulting in closed sharp feature lines that fit the segments such that the algorithm can be used as a pre-process step to find the areas where a surface patch can be defined. We are interested in point clouds from industrial applications, where closed sharp feature lines can be detected. It is not our goal to segment clouds with free form surfaces or fillets with a large radius. The algorithm differs from the existing feature line algorithms by the fact that it reconstructs

*kris.demarsin@cs.kuleuven.be

${ }^{\dagger}$ Metris N.V., Interleuvenlaan 86, B-3001 Leuven, Belgium 
closed sharp feature lines. The advantages of the algorithm are that (a) it is meshless, i.e. only the coordinates of the points are used, (b) it does not use curvature information, that is difficult to estimate in a noisy environment, (c) it intelligently clusters the points to create a graph that is much smaller than the original cloud, thus making it practical for large point clouds and, (d) it constitutes a pre-process step for surface reconstruction.

The algorithm is explained in the next section. In section 3 we illustrate some results of the algorithm applied to realistic point clouds, i.e. point clouds obtained from scanning industrial parts. We formulate the conclusions in section 4.

\section{Sharp feature line extraction algorithm}

\subsection{Algorithm overview}

Given a point cloud, we extract closed polygonal lines indicating the sharp edges. Algorithm 1 gives the different steps of the algorithm which will be explained in this section. We illustrate the algorithm with a point cloud representing two intersecting cylinders. The results of each step are depicted in figures 1,2 and 3, where the black lines approximate the sharp edges.

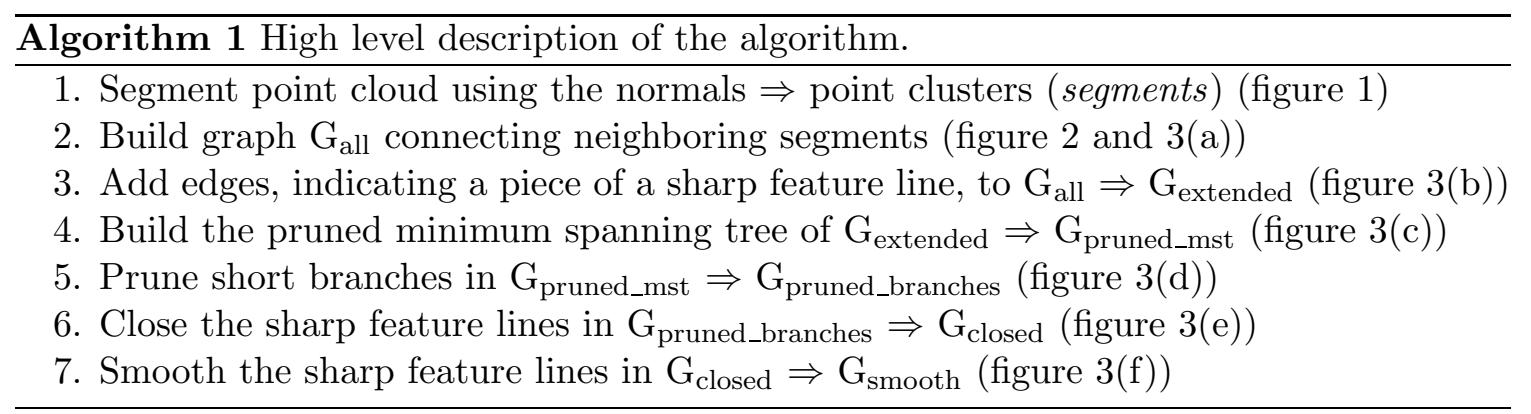

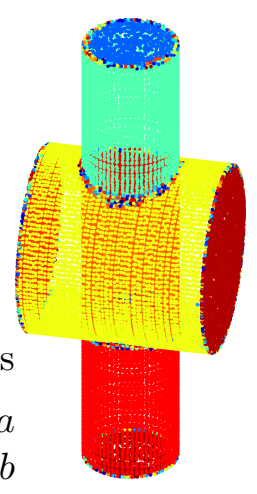

Figure 1: First order segmentation of two intersecting cylinders.

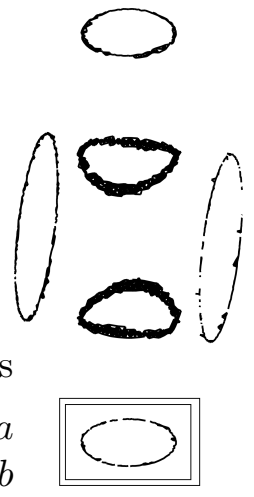

Figure 2: The graph $\mathrm{G}_{\text {all }}$; the area bounded by the rectangle is used to illustrate the following steps of the algorithm in detail.

\subsection{First order segmentation of point cloud}

Normal estimation and neighborhood selection The first step of the algorithm divides a point cloud in different clusters of points using a region growing method. For 


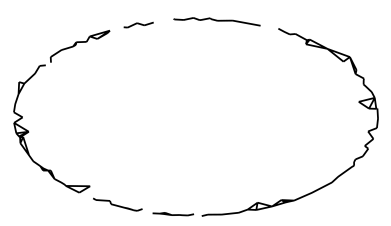

(a)

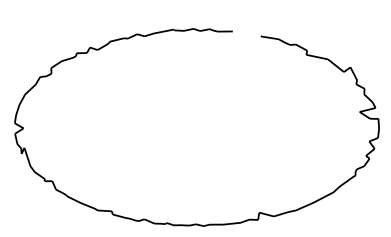

(d)

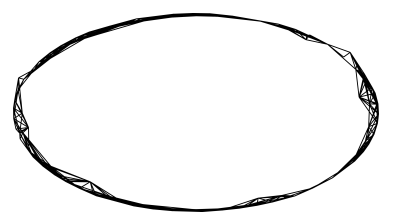

(b)

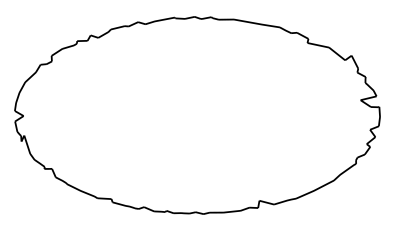

(e)

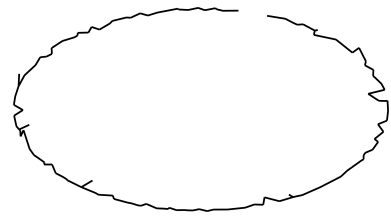

(c)

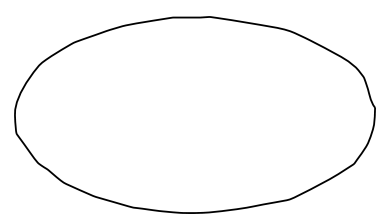

(f)

Figure 3: Result of each step of the algorithm illustrated with the detail of $G_{\text {all }}$ indicated by the rectangle in figure 2. (a) $G_{\text {all }}$ : graph of the segments; (b) $G_{\text {extended }}$ : $G_{\text {all }}$ with extra edges; (c) $G_{\text {pruned_mst }}$ : graph after building the pruned minimum spanning tree of $G_{\text {extended; }}$; (d) $G_{\text {pruned_branches }}$ : graph after pruning short branches in $G_{\text {pruned_mst }}$; (e) $G_{\text {closed }}$ : graph after closing the sharp feature lines in $G_{\text {pruned_branches }}$; (f) $G_{\text {smooth }}$ : graph after smoothing $\mathrm{G}_{\text {closed }}$.

that purpose, we need for every point the neighboring points as well as an estimation for the normal vector. In order to accurately detect the transition from a smooth area to a sharp edge, we estimate the normal vector as locally as possible. As we will see later on, to detect the sharp edges, we want the neighbors of a point $p$ to be distributed around $p$. With uniform sampling density, we could use the $k$ nearest neighbors, with $k$ small enough. However, in most realistic point clouds, this results in a neighborhood of $p$ located on one side, which is undesirable in our algorithm. If a triangular mesh is available, the 1-ring neighborhood of $p$ can be used to represent a good local neighborhood of $p$. Since we have a point cloud, we approximate this neighborhood by building a local mesh. We determine the $k$ nearest neighbors of $p$, with $k$ large enough, we construct the least squares plane through these points and we project the points on this plane. We then compute the Delaunay triangulation of these projected points and only the points that share an edge with $p$ in this triangulation, constitute the Delaunay neighborhood [1] of $p$, which we use as an approximation of the 1-ring neighborhood. The normal vectors are estimated by the PCA analysis of these 1-ring neighbors, as explained in [4].

Region growing We want to use an easy-to-implement region growing method to segment a point cloud based on the sharp edges using only normal estimation. Hence, we use a modification of the first order segmentation described by Vanco et al. ([9], [10], and [11]): we use one threshold angle which specifies the maximum acceptable angle between two adjacent normals in one segment. At a sharp edge, the normal estimation depends heavily on the computed 1-ring neighborhood, since this neighborhood is very local and these neighbors are located on both sides of the sharp edge. This means that the variation of the normals along a sharp edge is high, resulting in large segments with low variation of the normals bounded by small segments with high normal variation. Since these small 
segments indicate the sharp edges, we build a graph at segment level in the next step of the algorithm. Figure 1 illustrates the result of the first order segmentation, applied to the point cloud of the two intersecting cylinders, with each point colored corresponding to the segment it belongs to. Contrary to the method of Vanco et al. ([9], [10], and [11]), each cylindrical piece, bounded by sharp edges, consists of only one large segment with many small segments defining the boundary.

Perfectly aligned points Since the high normal variation is caused by differences in the 1-ring neighborhood, we have to be careful with point clouds where the points are perfectly aligned. Suppose we have a point cloud with uniformly distributed points on two planes, connected to each other in a right angle, as illustrated in figure 4. We will show that, even in this special case, there are many small segments at the sharp edge between the two planes after the segmentation. This is due to the high normal variation at the sharp edge, which has the following two causes. The first reason is the computation of the

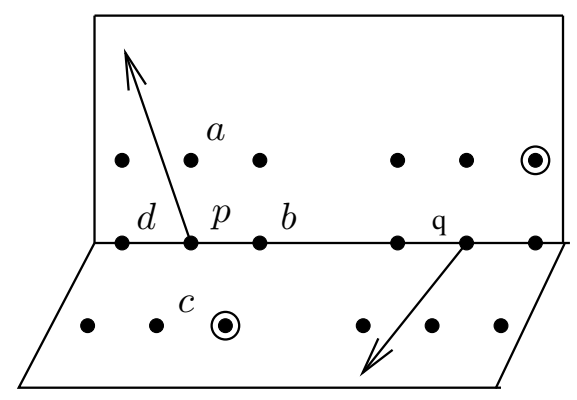

Figure 4: Detail of a point cloud with uniformly distributed points on two perpendicular planes. The two arrows are the normals of $p$ and $q$. The two circles indicate the chosen fifth point.

$k$ nearest neighbors. Suppose we want to compute the 5 nearest neighbors of the point $p$ on the sharp edge in figure 4. After selecting the points $a, b, c$ and $d$, which are located closest to $p$, we make a choice for the fifth point between the four other points close to $p$, since they are on the same distance from $p$. This choice depends on the order of the points in a data structure. This means that two distinctive points on the sharp edge, e.g. $p$ and $q$, can have unsimilar $k$ nearest neighbors and consequently, the computed Delaunay neighborhoods are incomparable. A small difference in this neighborhood results in a large difference in the computed normal. The second reason is as follows: suppose we do select equivalent $k$ nearest neighbors, then different Delaunay triangulations might be possible, which results again in different 1-ring neighborhoods and corresponding normals.

Note that taking $k=4$ results in the same normal for all the points at the sharp edge. However, this point cloud is very unrealistic, and even for such a point cloud, we are able to find a suitable $k$ to get high normal variation at the sharp edge.

\section{$2.3 \quad$ Building graph $\mathrm{G}_{\text {all }}$ of all segments}

Gumhold et al. [2] and Pauly et al. [7] introduced a graph approach to extract feature lines at the level of individual points. Since we are interested in sharp edges and the segmentation gives a strong indication of the location of these sharp edges, we base the algorithm on a 
graph structure at the level of segments, which yields cost reduction. Additionally, we keep information about neighboring segments and, contrary to [2] and [7], our prime focus is to detect closed sharp feature lines. Hence, we construct the connected graph $G_{\text {all }}$, where each vertex represents a segment and each edge connects two segments that contain at least one point with overlapping 1-ring neighborhood. For every vertex of the graph we keep a representation point of the segment, i.e. the average of all the points of the segment, and information about the size of the segment. Since the segmentation results in many small segments at the sharp edges we want to extract, we only plot the edges between two small segments to visualize this graph, see figures 2 and 3(a). Indeed, it are these edges that give us a first idea of the location of the sharp feature lines. However, remember that we keep the edges involving a large segment in the graph $G_{\text {all }}$ and they will be useful in the next step. From now on, we only process the graph and the point cloud is not needed anymore.

Since we have to distinguish between small and large segments, we could use a userdefined threshold. However, in practice, there are many small segments and less large segments with the large segments much larger than the small segments and thus taking the average of all sizes of segments is a good heuristic to separate the small segments from the larger ones.

\subsection{Adding edges to the graph $G_{\text {all }}$ yielding $G_{\text {extended }}$}

As already mentioned, our goal is to extract closed sharp feature lines, however, we see that the graph $G_{\text {all }}$ has already many unwanted 'gaps' between small segments. These gaps are caused by points on a sharp edge with a 'perfectly' estimated normal, i.e. the 1-ring neighborhood is located on one side of the sharp edge, due to non-uniform sampling density. The edges that close these gaps are part of the border between two large segments, i.e. these edges are located on a sharp edge and have to be included in the graph. For this purpose, we add edges to $G_{\text {all }}$ connecting two small segments that share two large neighboring segments.

This is illustrated in figure 5: due to the segmentation a small segment $s_{1}$ may exist that has only one small neighboring segment, namely $s_{2}$, and vice versa, which results in two unwanted gaps. We add the edge $\left(s_{3}, s_{1}\right)$ to $\mathrm{G}_{\text {all }}$ since $s_{3}$ and $s_{1}$ have two common large neighboring segments, namely $l_{1}$ and $l_{2}$. Similar, we add the edge $\left(s_{2}, s_{4}\right)$ resulting in

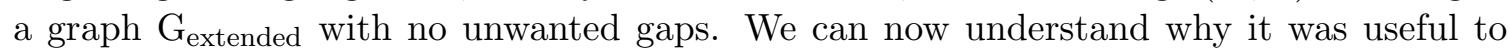
keep the edges involving a large segment in the graph $\mathrm{G}_{\text {all }}$ : the large neighboring segments are easily found. To avoid the addition of too many edges, e.g. in figure 5 we do not want $s_{1}$ to get connected with $s_{5}$, we only add edges with a euclidean distance between the two representation points which is less than the maximum euclidean distance between two small neighboring segments in $G_{\text {all }}$. The graph $G_{\text {extended }}$ for the detail of the two intersecting cylinders is illustrated in figure $3(\mathrm{~b})$. As with $\mathrm{G}_{\text {all }}$, we only plot the edges involving two small segments.

\subsection{Building $G_{\text {pruned_mst }}$, the pruned minimum spanning tree of $G_{\text {extended }}$}

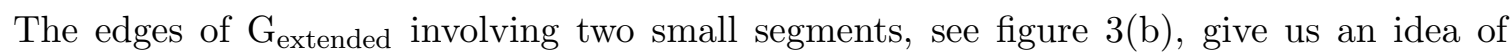
the location of the sharp feature lines. However, there are many cycles and therefor, we construct the minimum spanning tree (MST) of $\mathrm{G}_{\text {extended }}$. For this purpose, weights of edges between small segments are calculated as the distance between the representation points of the segments. Additionally, we attach large weights (larger than the weights between small segments) to edges involving a large segment. Building the MST of $\mathrm{G}_{\text {extended with these }}$ 


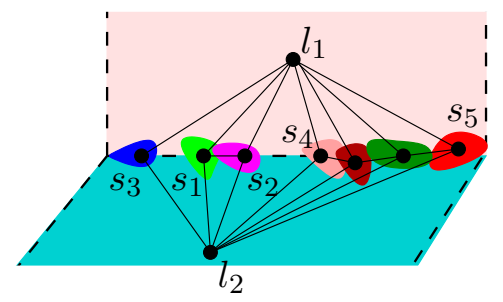

Figure 5: The graph $\mathrm{G}_{\text {all }}$ plotted on the segmentation of two perpendicular planes. Note that this time all the edges are plotted.

weights results in a graph with a reduced number of edges of which only a limited number of edges involve a large segment. We can now remove these latter edges which results in $\mathrm{G}_{\text {pruned_mst }}$, illustrated in figure $3(\mathrm{c})$.

\subsection{Construction of $G_{\text {pruned_branches }}$ by pruning short branches in $G_{\text {pruned_mst }}$}

Although constructing the pruned minimum spanning tree gives an initial reconstruction of the feature lines, the graph $\mathrm{G}_{\text {pruned_mst }}$ contains many short branches, as can be seen in figure 3(c). The next step of the algorithm, explained in section 2.7, reconstructs closed lines by linking each endpoint, i.e. a vertex in the graph with exactly one incident edge, with a suited point in the graph. For that reason, we prune the graph to remove unnecessary endpoints.

We use algorithm 2 to prune, which is a similar method as in [2]. Note that only the points with more than two incident branches are treated by the algorithm and no input of the user is needed to fine-tune the parameter $d_{\max }$ dependent on the point cloud. Setting the value of this parameter to 5 gave good results for all the tested point clouds. The resulting graph $\mathrm{G}_{\text {pruned_branches }}$ for the detail of the two intersecting cylinders can be seen in figure $3(\mathrm{~d})$.

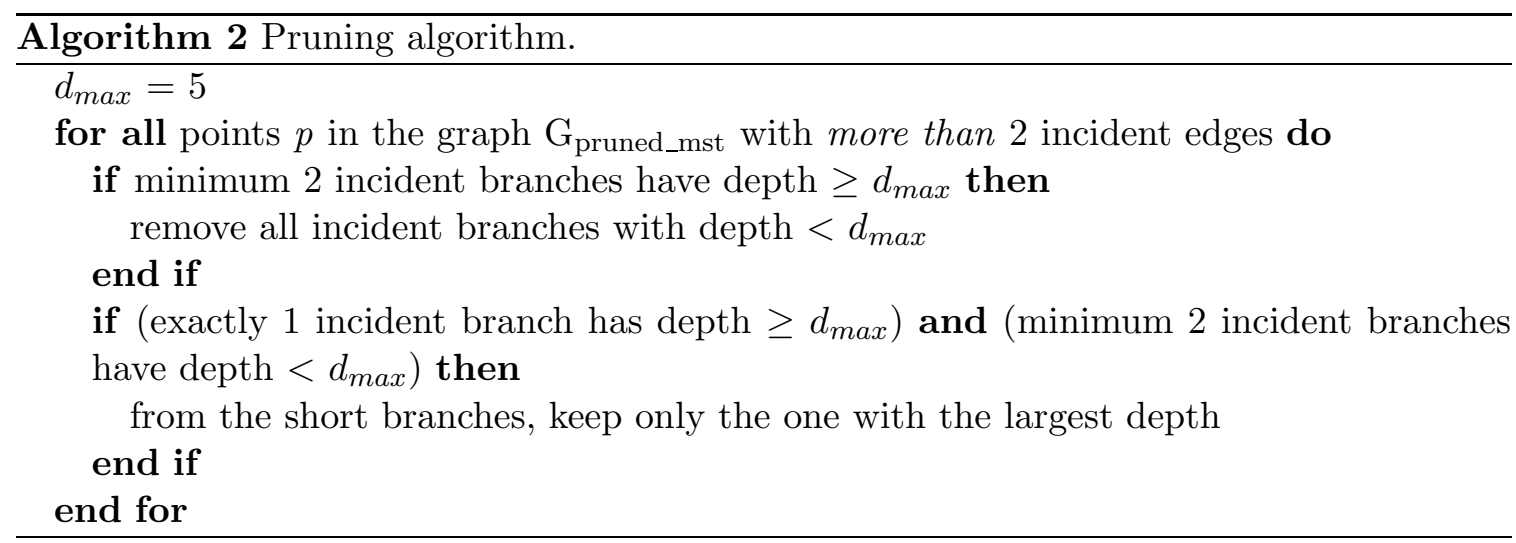




\subsection{Building $G_{\text {closed }}$ by closing the sharp feature lines in $G_{\text {pruned_branches }}$}

At this point, we have a graph $\mathrm{G}_{\text {pruned_branches }}$ with a reduced number of endpoints. Since our aim is to reconstruct closed lines, we introduce a 'connect' algorithm to link each endpoint with a suited point in the graph as follows: for each endpoint $p_{i}$ we compute the set $N$ of the $n$ nearest neighbors:

- Case 1: there exists at least one other endpoint in $N$ :

The endpoint $p_{i}$ is connected to the closest endpoint $q_{i}$ in $N$ which has no path with $p_{i}$ that is too short, i.e. the number of edges of each path is above a certain threshold $d$. In this way, we avoid generating small cycles, e.g. in figure 6 there exists a path between $p_{1}$ and $q_{1}$ which is too short and, consequently, we do not connect them. A good endpoint to link $p_{1}$ with is $q_{2}$ : they have only paths which are long enough. If no suitable endpoint is found in $N$, we do not link $p_{i}$ to anything, i.e. it stays an endpoint, like endpoint $p_{2}$ in figure 6 .

Since we have to search all the paths between $p_{i}$ and $q_{i}$ and because there can be many (long) paths, we optimize the algorithm by performing a depth first search with a restricted depth $d$, i.e. if at depth $d$ there is still no path found between $p_{i}$ and $q_{i}$, there are two possibilities: there is a path which is long enough (longer than $d$ ) or there is no path. In both cases, it is unnecessary to go deeper in the search tree, since no small cycles will be generated following the current path and we continue searching the other paths.

- Case 2: there exists no other endpoint in $N$ :

This case is similar to the previous case, but now we link $p_{i}$ with a point in $N$ which has more than one incident edge. For example $p_{4}$ is linked to $q_{4}$, but $p_{3}$ stays an endpoint, since all points in $N$ would generate a small cycle when connected with $p_{3}$.

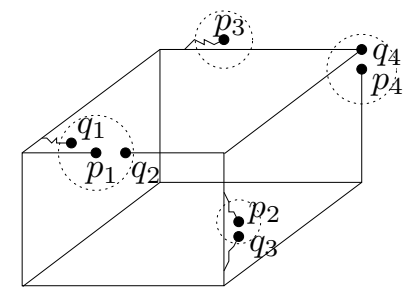

Figure 6: Illustration of the connect algorithm. The circles with $p_{i}$ as midpoint for $i \in$ $\{1,2,3,4\}$ indicate the spheres containing the $n$ nearest neighbors of $p_{i}$.

If, after the connect algorithm, there still exist endpoints, we remove all the edges starting from each endpoint until a point with more than two incident edges is reached, e.g. in figure 6 the four short branches ending in respectively $p_{2}, q_{3}, q_{1}$ and $p_{3}$ will be pruned. The connect algorithm can be seen as a last clean up step: 'noisy' branches, which are restricted to a minimum because of the previous steps of the algorithm, are pruned and all sharp feature lines are closed.

The value of $n$ must be chosen carefully to ensure that the needed points to link with are in $N$ and to avoid connections with points that are located too far. The value of $d$ must be chosen such that all the sharp feature lines are extracted and all noisy branches are removed. 
The two endpoints that still existed in figure 3(d) are now connected, as illustrated in figure $3(\mathrm{e})$.

\subsection{Generating $\mathrm{G}_{\text {smooth }}$ by smoothing $\mathrm{G}_{\text {closed }}$}

The method we use to get a smooth graph $\mathrm{G}_{\text {smooth }}$, see figure 3(f), is explained in [12] .

\section{Results}

To illustrate the different steps of the algorithm, we only showed a detail of the two intersecting cylinders. The whole graphs $\mathrm{G}_{\text {closed }}$ and $\mathrm{G}_{\text {smooth }}$ are illustrated in figures $7(\mathrm{a})$ and $7(\mathrm{~b})$. The results for a cube can be seen in figure 8: we see how the connect algorithm results in perfectly closed sharp feature lines. As with the cylinders, we only plot the edges involving two small segments. Figure 9 illustrates the final result for a detail of a mobile phone, a typical example of a point cloud used in industrial applications. This point cloud has been generated by a laser scanner and thus some noise is present. We note that the algorithm does not guarantee good results when two sharp feature lines are located too close to each other depending on neighborhood selection and point density: there is an unwanted gap at the right caused by the pruning, and there exist some edges connecting two sharp feature lines. One way to partially solve the latter problem is as follows: in the connect algorithm, we could generate an ellipsoid in the direction of the sharp feature line instead of a sphere to find the neighboring points to connect to.

The final result for a larger part of a mobile phone, also real data from a scanner, is illustrated in figure 10. Contrary to the previous point clouds, this point cloud does not respresent a solid, i.e. it has a boundary which we extract and include in the graph. By comparing the final graph with the segmentation, we see how well the detected lines fit the segmentation, but we see also that a few sharp feature lines are not detected because they consist of cycles which are too short. Additionally, where two lines are located too close to each other, they are extracted as one line.

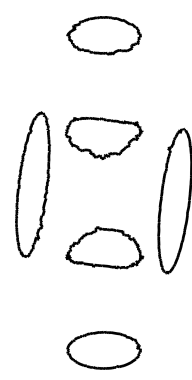

(a)

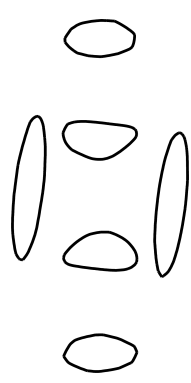

(b)

Figure 7: Results for the two intersecting cylinders. (a) $G_{\text {closed }}$; (b) $G_{\text {smooth }}$.

Table 1 presents information about the segmentation, e.g. because of the high normal variation at the sharp edges, the average size of a small segment is close to unity. In the case of the cube and the cylinders, we see that the segmentation results in the correct number of large segments: the segmentation fits the extracted sharp feature lines perfectly. Table 2 gives for every step of the algorithm the number of vertices and edges of the corresponding 


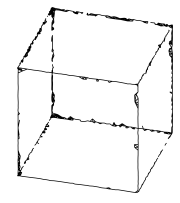

(a)

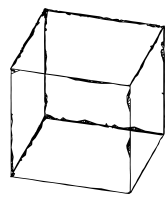

(b)

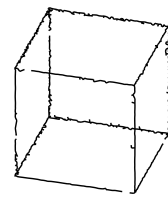

(c)

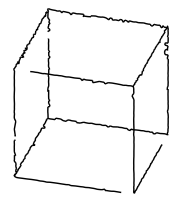

(d)

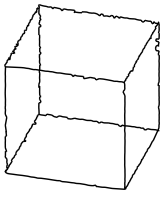

(e)

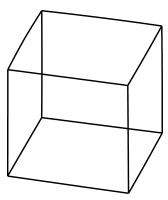

(f)

Figure 8: Result of each step of the algorithm applied to the cube. (a) $G_{\text {all }}$ : graph of the segments; (b) $G_{\text {extended }}: G_{\text {all }}$ with extra edges; (c) $G_{\text {pruned_mst }}$ : graph after building the pruned minimum spanning tree of $G_{\text {extended }}$; (d) $G_{\text {pruned_branches }}$ : graph after pruning short branches in $\mathrm{G}_{\text {pruned_mst }}$; (e) $\mathrm{G}_{\text {closed }}$ : graph after closing the lines in $\mathrm{G}_{\text {pruned_branches }}$; (f) $\mathrm{G}_{\text {smooth }}$ : graph after smoothing.

graph. In the case of the two intersecting cylinders, we start with a point cloud of 26846 points and then we build a graph $\mathrm{G}_{\text {all }}$ of 1706 vertices and 6086 edges. After adding edges to $G_{\text {all }}$, every following step reduces the memory consumption of the graph: a huge reduction in the number of edges happens when building $G_{\text {pruned_mst }}$ and $G_{\text {pruned_branches }}$. In general, in the close step, more edges are removed than added, since noisy branches are pruned. Note that for the large mobile phone point cloud the boundary is included just before the close step. The table also illustrates the time consumption of the algorithm. The segmentation step requires more time compared to the other steps of the algorithm, because this step has to grow through all the points of the point cloud and the normal for each point needs to be estimated. We could make the segmentation much faster by estimating the normal as the normal of the least squares plane through the $k$ nearest neighbors. However, for realistic point clouds, a small $k$ might generate neighbors only on one side of the sharp edge causing no small segments, and a large $k$ makes it impossible to accurately detect the transition from a smooth surface to a sharp edge.

The algorithm uses some parameters, however, most of these parameters have the same value for all tested models: $k$ of the $k$ nearest neighbors needed for normal estimation, the threshold angle in the segmentation step and $d_{\max }$ in the pruning step. Only a few parameters need to be specified by the user, namely $n$ and $d$ in the connect algorithm. In practice, this choice is not that hard and trying a few parameters is not time consuming since the connect algorithm is not expensive and can run separately from the previous steps.

Table 1: Information about the segmentation for the different point clouds.

\begin{tabular}{lllll}
\hline & $\begin{array}{l}\text { Size of } \\
\text { point cloud }\end{array}$ & $\begin{array}{l}\text { Average size } \\
\text { small segments }\end{array}$ & $\begin{array}{l}\text { Number of } \\
\text { small segments }\end{array}$ & $\begin{array}{l}\text { Number of } \\
\text { large segments }\end{array}$ \\
\hline Phone small & 11034 & 1.24 & 1610 & 37 \\
Cube & 15206 & 1.17 & 851 & 6 \\
Cylinders & 26846 & 1.11 & 1696 & 10 \\
Phone large & 110053 & 1.62 & 5247 & 69 \\
\hline
\end{tabular}




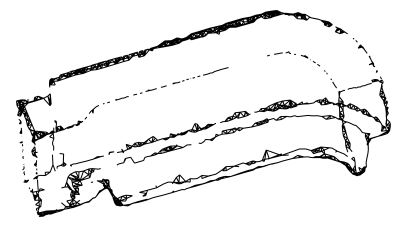

(a)

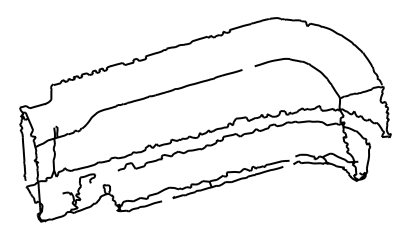

(d)

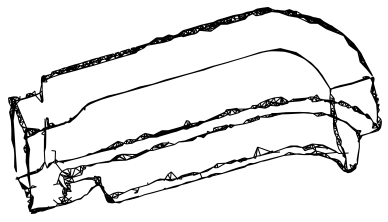

(b)

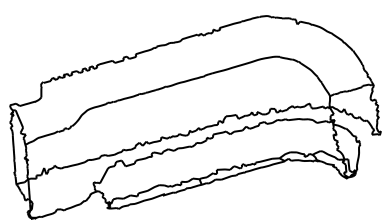

(e)

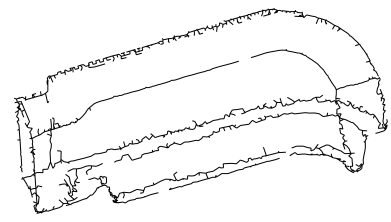

(c)

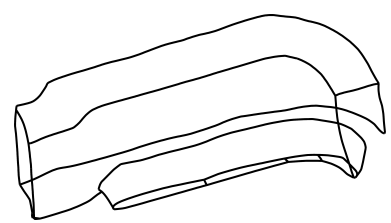

(f)

Figure 9: Result of each step of the algorithm applied to the mobile phone point cloud. (a) $\mathrm{G}_{\text {all }}$; (b) $\mathrm{G}_{\text {extended }}$; (c) $\mathrm{G}_{\text {pruned_mst }}$;

(d) $G_{\text {pruned_branches }}$;

(e) $\mathrm{G}_{\text {closed }}$;

(f) $\mathrm{G}_{\text {smooth }}$.

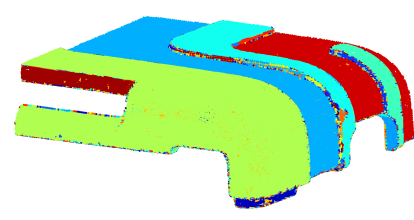

(a)

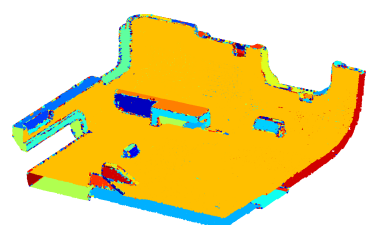

(b)

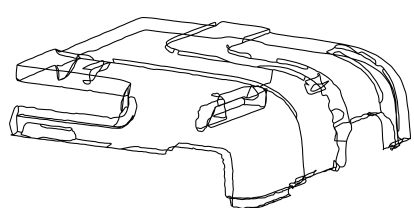

(c)

Figure 10: Results for a larger detail of a mobile phone. (a) and (b) illustrate two different views of the segmentation; (b) $\mathrm{G}_{\mathrm{smooth}}$.

\section{Conclusion and future work}

We presented an algorithm to extract sharp edges from a point cloud without estimating the curvature and without triangulating the point cloud. Additionally, all extracted lines are closed at the end of the algorithm. We start with a very simple region growing method with well chosen normals, resulting in an initial segmentation based on the sharp edges. Afterwards, we build and manipulate a graph of the segments. Using a graph structure at the level of segments yields faster execution times and less memory consumption, making the algorithm suitable for large point clouds. Once we build the graph of the segments, the point cloud is not needed anymore and we only need to process the graph in the following steps: adding extra edges, construction of the minimum spanning tree, pruning, closing and smoothing the sharp feature lines.

Although the segmentation step is time consuming for large point clouds, together with the closed lines, it constitutes a pre-process step in finding a curve network. In the future, we plan to construct this network, which consists of a set of loops, where each loop defines 
Table 2: Complexity and time consumption of the different steps. The segmentation is decomposed in two steps: the normal estimation $(\vec{n})$ and the region growing (RG). The timings are in seconds and generated on an Intel Pentium 4, 3.20 GHz.

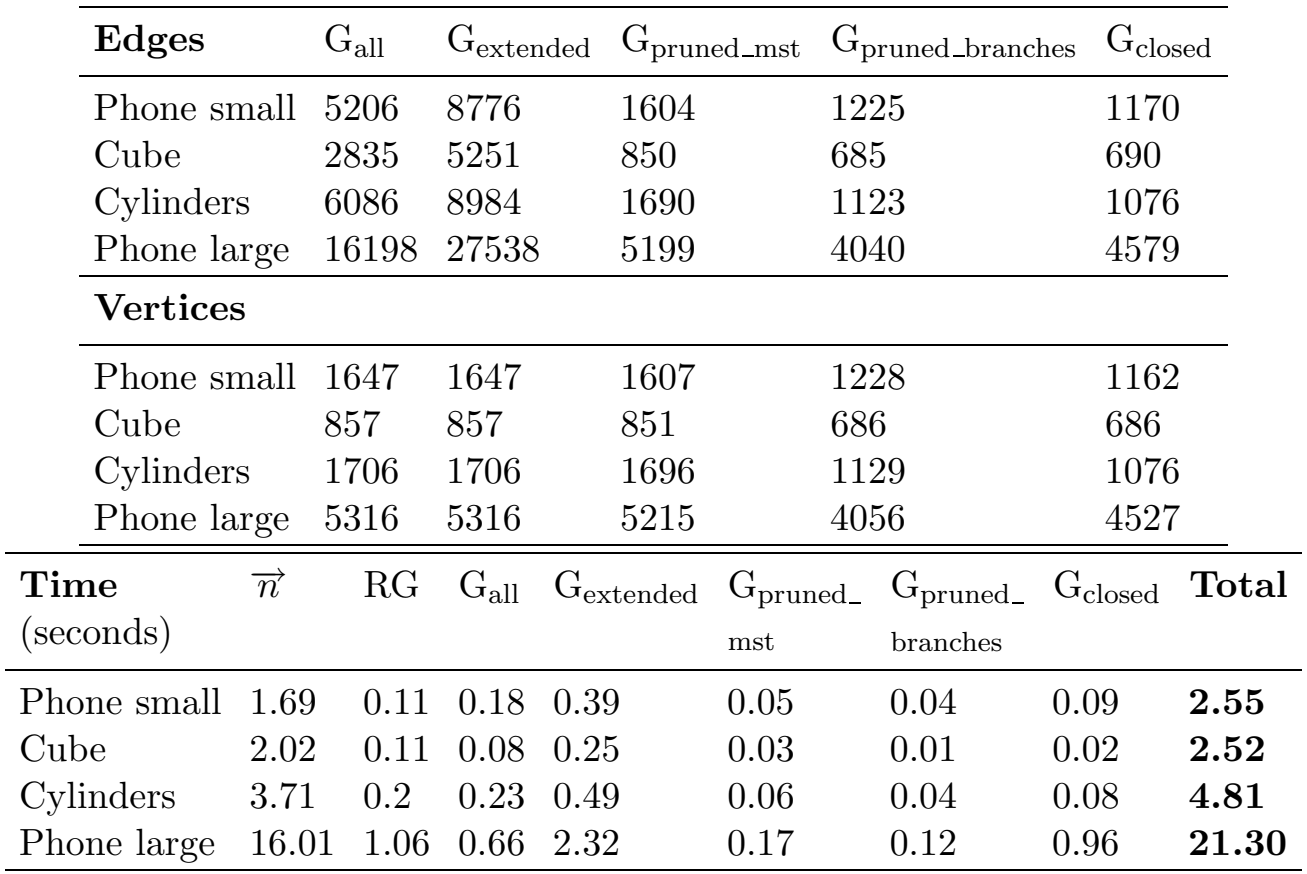

the boundary of an area where a patch can be fitted. When all segments bounded by sharp edges are known, in a next step, we can continue with each segment individually, e.g. to detect also tangent continuous but curvature discontinuous features like fillets.

\section{Acknowledgements}

The two mobile phone point clouds are courtesy of Metris N.V. Belgium.

\section{References}

[1] M. S. Floater and Martin Reimers. Meshless parameterization and surface reconstruction. Computer Aided Geometric Design, 18(2):77-92, 2001.

[2] S. Gumhold, X. Wang, and R. MacLeod. Feature extraction from point clouds. Proceedings of the 10th International Meshing Roundtable, pages 293-305, 2001.

[3] Klaus Hildebrandt, Konrad Polthier, and Max Wardetzky. Smooth feature lines on surface meshes. Symposium on Geometry Processing, pages 85-90, 2005.

[4] K. Hormann. Theory and Applications of Parameterizing Triangulations. PhD thesis, Department of Computer Science, University of Erlangen, November 2001.

[5] Yutaka Ohtake and Alexander Belyaev. Automatic detection of geodesic ridges and ravines on polygonal surfaces. The Journal of Three Dimensional Images, 15(1):127$132,2001$. 
[6] Yutaka Ohtake, Alexander Belyaev, and Hans-Peter Seidel. Ridge-valley lines on meshes via implicit surface fitting. SIGGRAPH, pages 609-612, 2004.

[7] Mark Pauly, Richard Keiser, and Markus H. Gross. Multi-scale feature extraction on point-sampled surfaces. Comput. Graph. Forum, 22(3):281-290, 2003.

[8] G. Stylianou and G. Farin. Crest lines extraction from 3D triangulated meshes. Hierarchical and Geometrical Methods in Scientific Visualization, pages 269-281, 2003.

[9] M. Vanco, G. Brunnett, and Th. Schreiber. A direct approach towards automatic surface segmentation of unorganized 3D points. Proceedings Spring Conference on Computer Graphics, pages 185-194, 2000.

[10] Marek Vanco and Guido Brunnett. Direct segmentation for reverse engineering. In Proceedings International Symposium on Cyber Worlds, pages 24-37, 2002.

[11] Marek Vanco and Guido Brunnett. Direct segmentation of algebraic models for reverse engineering. Computing, 72(1-2):207-220, 2004.

[12] T. Volodine, D. Vanderstraeten, and D. Roose. Smoothing of meshes and point clouds using weighted geometry-aware bases. Report TW 451, Department of Computer Science, K.U.Leuven, Leuven, Belgium, March 2006.

[13] Kouki Watanabe and Alexander G. Belyaev. Detection of salient curvature features on polygonal surfaces. Computer Graphics Forum, 20(3):385-392, 2001. 